\title{
Aplicación de interferometría radar en el estudio de subsidencias en el Valle de Toluca, México.
}

Dávila-Hernández, Norma Angélica y Madrigal-Uribe, Delfino

\section{Resumen:}

El acuífero del Valle de Toluca pertenece a la cuenca alta del Río Lerma, en el Estado de México, siendo considerado como el segundo acuífero más sobre-explotado en México. Actualmente, la extracción del recurso ha causado impactos negativos como los procesos de subsidencia, dando lugar a agrietamientos tanto en zonas urbanas como industriales, implicando así la compactación de los estratos geológicos en la zona de estudio, que afectan al terreno.

El objetivo fue evaluar procesos de subsidencia en el Valle de Toluca, México, a partir de Técnicas de Interferometría Diferencial que permitan la estimación precisa de la velocidad de deformación del terreno.

Para ello se aplicó el método de Interferometría de Radar (InSAR) de mayor precisión conocido como "Persistent Scatterer" (PS). A diferencia de DinSAR, PS permite la selección de pixeles que presentan una mayor calidad en su amplitud y fase (bright radar targets) a partir de multiples datos SAR. Se adquirieron así 30 imágenes ENVISAT-ASAR en modo Single Light Complex (SLC) para el periodo 2003-2010, con una resolución rango-acimut de 20.16 x 24.34, respectivamente.

Los resultados muestran velocidades de deformación superiores a los $50 \mathrm{~mm} / a n ̃ o$

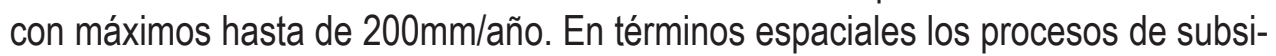
dencia máximos corresponden a la parte central de la cuenca, coincidiendo con el casco histórico de la Ciudad de Toluca, al igual que hacia el este de la cuenca en zonas de cultivo y humedales. Para la validación del trabajo se analizó la disminución del nivel superficial del acuífero a partir de datos piezométricos y su relación estratigráfica en campo. Con base en lo anterior, se encontró una relación directa entre las zonas afectadas por procesos de subsidencias de acuerdo con PS y los puntos registrados con mayor abatimiento del acuífero en relación con la profundidad del nivel estático, referido al terreno. 
Palabras clave: subsidencias, interferometría, radar diferencial, México

\section{Abstract:}

The Toluca Valley Aquifer belongs to High Lerma River Basin in Mexico State, showed the second most over exploited aquifer of Mexico. At present, the resource extraction had caused negative impacts like subsidence processes whose induce fissures as many urban zones as industrial zones, thus involving a geological and soil compression in the design zone.

The objective was to evaluate subsidence processes in the Toluca Valley, since interferometric differential techniques who enable to precisely estimation of the land velocity deformation.

In fact it applied the radar interferometric method (InSAr) well-known like "Persistent Scatterer" (PS), to major precision. On difference of DinSAR, PS allows pixels selection who shows major quality in his amplitude and phase (bright radar targets) beginning from manifolds SAR dates. Were obtained 30 ENVISAT-ASAR images thus in Single Light Complex (SLC) mode, for 2003-2010 period, with rank-acimut resolution of $20.16 \times 24.34$.

The results show deformation velocities upper $50 \mathrm{~mm} / \mathrm{year}$ with great limits as many as to $200 \mathrm{~mm} / \mathrm{year}$. In spatial terms, the great subsidence processes correspond to the central basin zone, coinciding with historic center of Toluca city, like than east of basin in zones cultivation and swamps. For work validation it analyzed the superficial aquifer decrease level since piezometric dates and her stratigraphic relationship in field. Whatever, it found a direct relationship between affected zones of subsidence in agreement with PS and registered points with major aquifer low spirits in record with depth static level of ground.

Keywords: Subsidences, Interferometry, differential radar, Mexico

Dávila-Hernández, Norma Angélica (nadavilah@uaemex.mx); Madrigal=Uribe, Delfino. Facultad de Geografía de la UAEM. Cerro de Coatepec s/n, Ciudad Universitaria, CP 50110. 


\section{INTRODUCCIÓN}

El acuífero del Valle de Toluca (AVT) se localiza en la cuenca superior del Río Lerma en México Central (Figura 1) cubriendo una extensión de 2,117.82 Km². Actualmente es considerado uno de los acuíferos más sobre-explotados del país, lo anterior se debe a la inexistente planeación histórica en la preservación de los recursos hídricos, aunado a una excesiva demanda de los mismos por parte de la población ubicada en la zona central de nuestro país. A partir de 1942 se comenzó la perforación de pozos en la zona de estudio con el objeto principal de proveer de agua a la Ciudad de México y en un segundo plano a la Ciudad de Toluca siendo esta última la ciudad más importante de la zona de estudio.

Actualmente se encuentran operando más de 300 pozos de extracción, los cuales proveen el $30 \%$ del agua potable consumida en el Valle de México, mientras que únicamente $2 \%$ del total de volumen extraído es destinado para su consumo en la Ciudad de Toluca y comunidades aledañas; por consiguiente, la sobre-explotación del AVT ha dado lugar a la compactación de los estratos geológicos (secuencia de depósitos volcanoclásticos dacíticos-andesíticos y lacustres principalmente), lo que propicia la formación de patrones de fracturas asociadas a subsidencias de tierra y en algunos casos hasta el desarrollo de procesos de colapsamiento del suelo. En términos ecológicos la sobre-explotación del acuífero ha provocado una extinción gradual de lagos y humedales ubicados al este de la cuenca, los cuales se encuentran en una de las zonas más importantes de recarga (Garfias et al., 2008). 
Figura 1. Mapa de subsidencias del terreno utilizando el método PS (Persistent Scatterer)

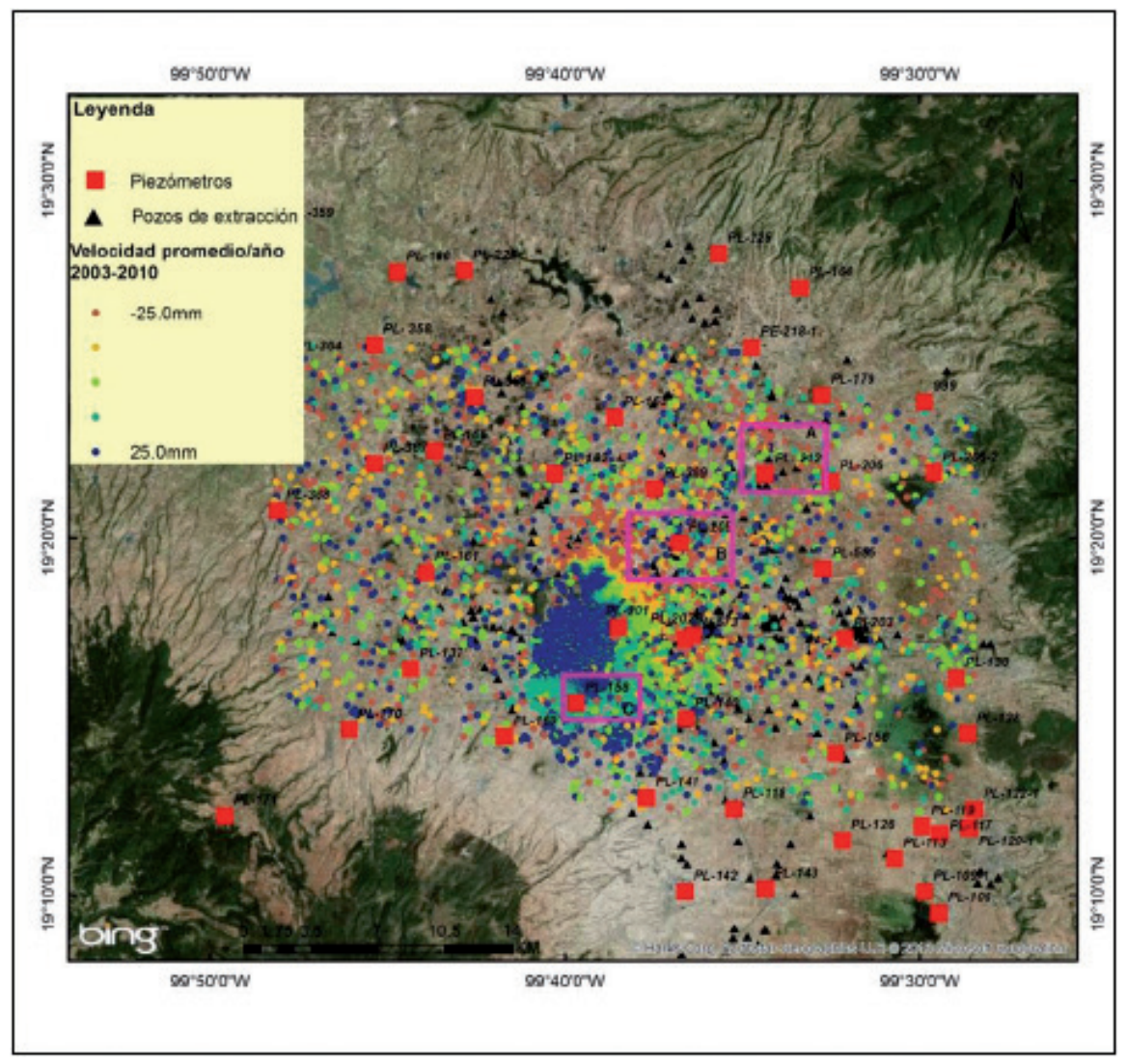

Fuente: Elaboración propia a partir de imágenes ENVISAT 2003-3010

La Percepción Remota guarda una estrecha relación en el estudio de cualquier fenómeno natural o social ocurrido en el espacio geográfico, ya que permite su evaluación y monitoreo a través del tiempo. Tal es el caso de la aplicación de técnicas de radar como la Interferometría Diferencial (DinSAR), llegando a ser una herramienta poderosa en la identificación de patrones espaciales en la superficie terrestre asociados a una variación vertical y horizontal con respecto a un determinado periodo de tiempo. De esta forma, DinSAR permite la estimación a escala centimétrica de cualquier desplazamiento vertical en dirección de radar a partir de la estimación de cambio de fase entre dos imágenes de radar tomadas en tiempos distintos. Persistent Scatterer (PS), a diferencia de la técnica convencional DinSAR de pares individuales, permite utilizar en un mismo tiempo múltiples imágenes a 
partir de la selección de pixeles que preservan la coherencia y fase a través del tiempo permitiendo una estimación milimétrica de la razón de cambio del fenómeno estudiado; siendo una técnica eficiente en el estudio de subsidencia en áreas urbanas principalmente (Herrera et al., 2009; Colesanti et al., 2003; López et al., 2009; Chen et al., 2012; Calderhead et al., 2012).

El objetivo del presente estudio radica en la evaluación multi-temporal 20032010 de subsidencias de tierra en el ATV con énfasis en la Ciudad de Toluca y zonas semi-urbanas aplicando la técnica PS utilizando imágenes de radar de mediana resolución Evisat-ASAR. El presente estudio muestra los resultados preliminares de la investigación así como sus alcances y retos futuros.

\section{METODOLOGÍA}

La Interferometría Diferencial convencional estima la diferencia de fase a partir de dos adquisiciones de datos radar de distintas fechas; sin embargo, factores asociados a líneas de base normales superiores a los 1000m, cambios físicos en el terreno al adquirir la escena en un tiempo determinado y heterogeneidad atmosférica, así como líneas de base multitemporales mayores, contribuyen a la decorrelación de la información entre pares interferométricos complejos para un adecuada resolución de la fase. De esta forma, Persistent Scatterer (PS) es un método derivado de DinSAR convencional donde a partir del análisis de amplitud de la imagen y la generación posterior de interferogramas, permite seleccionar pixeles candidatos cuya amplitud y fase haya sido conservada a través del tiempo. Particularmente, en la investigación se utilizó el método de dispersión normalizada de la amplitud (Ferreti et al., 2000):

$$
\operatorname{Dan}=\frac{\rho a}{\mu a}
$$

Donde, Dan representa la dispersión de amplitud normalizada para cada pixel seleccionado, el cual es determinado por el radio entre la dispersión de la amplitud $\rho a$ y la media de las amplitudes $\mu a$ para un periodo de tiempo; de forma tal que, un valor alto en la dispersión de la amplitud se asocia a una pérdida de la fase interferométrica.

De esta forma se logra estimar la fase atmosférica de manera precisa tomando ventaja de la correlación de la información asociada a la vecindad de los 
pixeles seleccionados (Sousa et al., 2010). Es importante señalar que este tipo de técnicas tiene una considerable utilidad para mediciones DinSAR en áreas urbanas, debido principalmente a la configuración de los edificios y la infraestructura urbana, la cual en términos de retrodispersión de la señal de radar (scattering) no presenta fluctuaciones.

En la presente área de estudio se han realizado estudios preliminares de subsidencias de tierra utilizado técnicas convencionales DinSAR (see Calderhead et al., 2010 and Calderhead et al., 2012) de acuerdo a lo antes descrito. En su lugar, la investigación propuso utilizar el método PS por ser una técnica que permite obtener una mayor exactitud (milímetros/año) en las mediciones de deformaciones verticales de la superficie LOS (Line of Sight); logrando así estimar velocidades de deformación del orden mm/año para un periodo de tiempo determinado. Para ello, se utilizaron 30 imágenes ENVISAR-ASAR para el periodo 2003-2010 en órbita descendente, (faltan otros datos).

Para la estimación de la interferometría diferencial multitemporal es necesario seleccionar una imagen de referencia, la cual debe corresponder con la línea de base multitemporal mayor con respecto al resto de las imágenes; así se seleccionó la imagen de radar con fecha 03/07/2013 con una línea de base temporal de 3650 días. De igual manera, en el procesamiento de pares interferométricos utilizados como base para la generación de interferogramas, se consideraron todas aquellas líneas de base normales cuyo límite mínimo y máximo oscilara entre $15 \%$ y $80 \%$; además de considerar un límite en coherencia de 0.80 . De esta forma, se obtuvieron un total de 66 combinaciones para la generación de interferogramas diferenciales, para los cuales se utilizó el modelo digital de elevación SRTM 3, con una resolución de 30 metros, con el objeto de remover la fase asociada a la topografía original del terreno.

Como forma de corroborar los resultados de las subsidencias se hizo una correlación directa con los datos aportados por los piezómetros de la Comisión Nacional del Agua para las mismas fechas y los mismos lugares en los que a su vez se realizaron mediciones con GPS y estaciones totales, mediante trabajo de campo durante el año 2012 y 2013. De la misma manera se midieron, fotografiaron y se registraron afectaciones sensibles en el terreno como los agrietamientos, la generación de zanjas y la presencia de daños en construcciones y propiedades, ubicándolos posteriormente en mapas e imágenes satelitales de tipo multiespectral. 
Con el fin de explicar resultados se obtuvieron datos de investigaciones anteriores (Madrigal et al. 2010; Díez 2010) sobre morfoestructuras tectónicas que circundan y atraviesan el Valle de Toluca, ubicándolas en las imágenes de radar, como forma de relación y de explicación de la concentración de los procesos de subsidencia, asociados a procesos de crecimiento urbano y de zonas industriales, así como a la demanda y la extracción de agua del subsuelo.

\section{RESULTADOS}

A partir de DinSAR convencional, PS permite estimar velocidades promedio del orden de mm/año. En el mapa de la Figura 1, se muestra las velocidades LOS promedio para el periodo 2003-2010, donde pixeles estables se observan hacia el casco histórico de la ciudad, lo cual se encuentra relacionado con una mayor densidad de infraestructura urbana y también con accidentes tectónicos locales y regionales. A partir de $3.5 \mathrm{~km}$ (con respecto a los pixeles estables) en dirección norte, noreste se observan las mayores concentraciones de movimientos diferenciales con velocidades máximas de hasta $27 \mathrm{~mm} /$ año y velocidades promedio de $18 \mathrm{~mm} /$ año. Finalmente a partir de $3.0 \mathrm{~km}$ (con respecto a los pixeles estables) hacia el este se distribuyen los pixeles asociados a movimientos diferenciales medios con veloci-

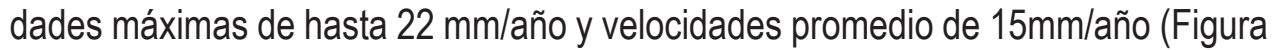
1).

Con la intención de ejemplificar los movimiento diferenciales en la zona de estudio a través del tiempo, se seleccionaron tres áreas representativas de cambios espaciales en términos de LOS de acuerdo al mapa generado. Dichos datos fueron comparados con datos piezométricos históricos los cuales muestran el nivel estocástico del agua y donde cada uno de ellos cuenta con al menos 4 sensores a distinta profundidad. Para este trabajo se seleccionaron profundidades superficiales menores a $30 \mathrm{~m}$ y profundidades mayores a los $130 \mathrm{~m}$, en los que los piezómetros fuera de los límites mencionados no fueron considerados. Los valores fueron obtenidos de los datos de la Comisión Nacional del Agua para el período 2000-2010.

En las figura 2, 3 y 4 se muestran tres gráficas de las áreas seleccionadas de acuerdo al mapa de la figura 1, cuya selección se definió en función de los límites de cambio LOS de acuerdo a su distribución y su cercanía con los piezómetros que cumplieran con las condiciones antes señaladas. Así, en concordancia con la Figura 
2, los gráficos A y B que corresponden a la zona norte-noreste del mapa de la Figura 1 , presentan las mayores deformaciones diferenciales del terreno de acuerdo a los valores de LOS, mientras que la gráfica $\mathrm{C}$ representa una zona estable (es decir, donde LOS presenta movimientos negativos).

Figura 2. Grafica de la zona A con respecto al mapa de la Figura 1.

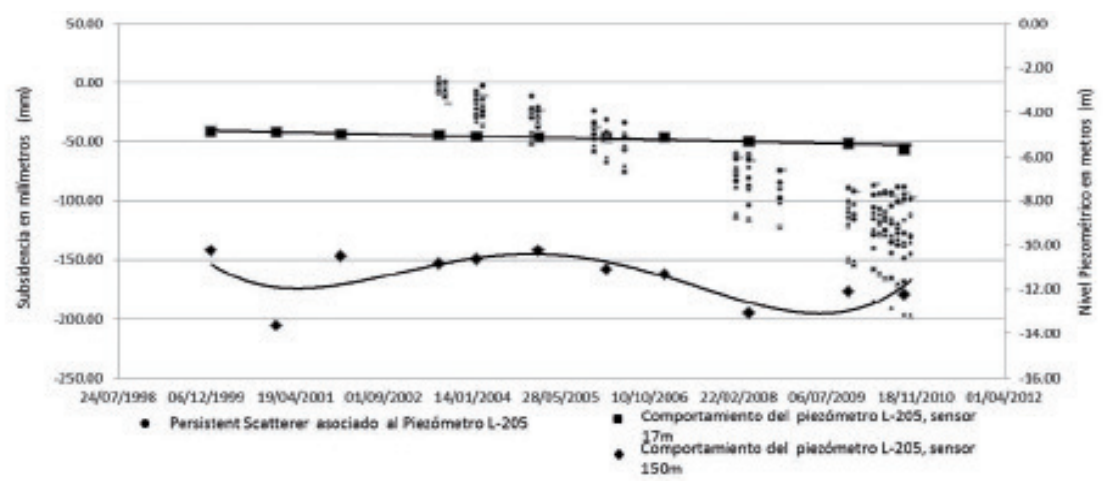

Fuente: Elaboración propia con datos piezométricos de CONAGUA 1998-2012

Como puede observarse también en las gráficas de la figuras 2 y 3 , tanto en A como en B el nivel piezométrico del agua en relación al sensor superficial presenta una razón de cambio no mayor a 5 metros, mientras que el sensor profundo muestra un cambio negativo para el periodo 2000-2010, lo cual se identifica con un abatimiento máximo de 40 metros de acuerdo al nivel de agua del acuífero. Esto a su vez guarda una relación directa en superficie de acuerdo a las velocidades LOS (puntualizadas en el párrafo anterior) de los pixeles seleccionados cercanos a los piezómetros L-205 yL-1158 respectivamente, presentando velocidades promedio de $26 \mathrm{~mm} / \mathrm{año.}$ 
Figura 3. Gráfica de la zona B con respecto al mapa de la Figura 1.

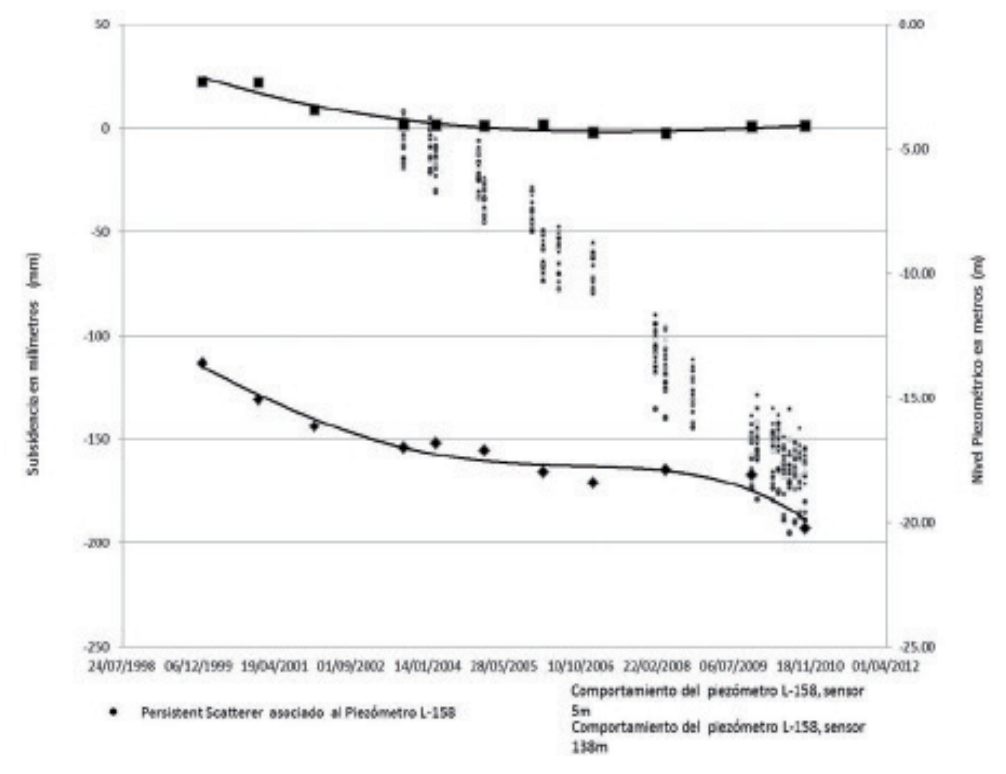

Fuente: Elaboración propia con datos piezométricos de CONAGUA 1998-2012

Caso contrario sucede con el piezómetro L-212 (grafica C, Figura 4) el cual se ubica en una zona donde los valores LOS son estables, esto se demuestra observando como los pixeles seleccionados reportan valores positivos con respecto al tiempo, lo que se encuentra respaldado por el comportamiento de los sensores piezométricos tanto a nivel somero como a nivel superficial, el cual es prácticamente estable para los 10 años considerados. 
Figura 4. Grafica de la zona C con respecto al mapa de la Figura 1.

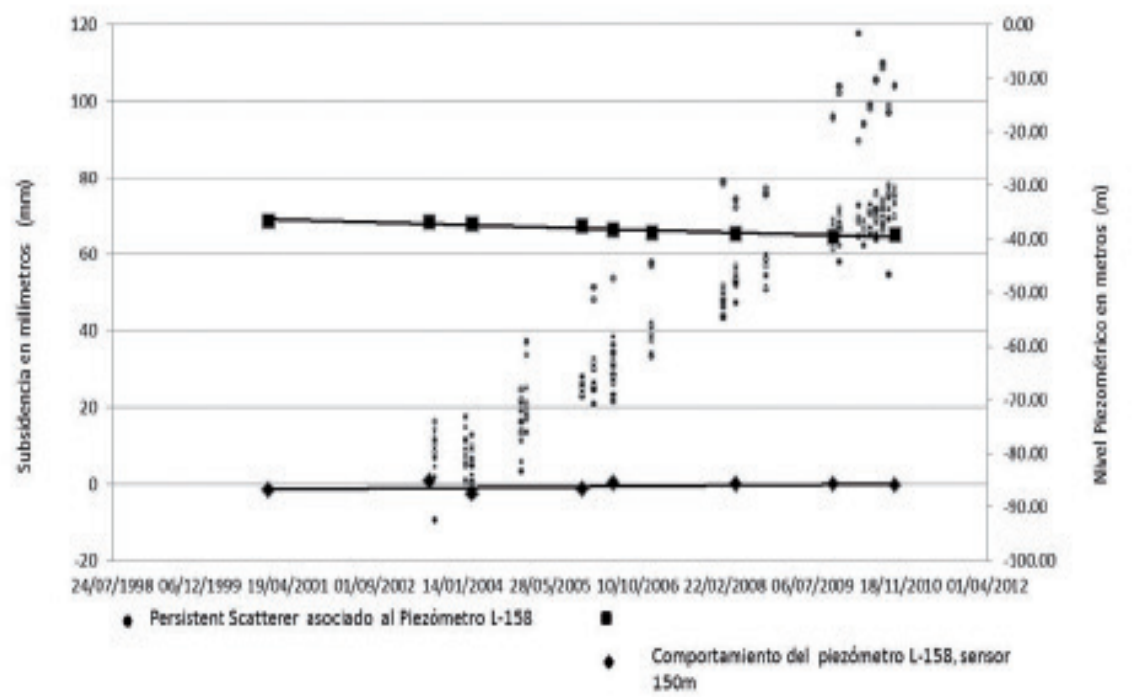

Fuente: Elaboración propia con datos piezométricos de CONAGUA 1998-2012

Los procesos de subsidencia en el Valle de Toluca responden a la conjunción de varios aspectos que son característicos de su geología, su morfología, su comportamiento hidrológico y geohidrológico y los patrones de asentamiento urbano e industrial que conforman la demanda y la extracción de agua subterránea. Aunque se maneja en las referencias bibliográficas antecedentes mucho de ese comportamiento, los resultados encontrados demuestran muchas asociaciones importantes que deben destacarse para explicar en gran medida la ubicación de las zonas críticas en los procesos de subsidencia.

Esto entraña el destacar y dimensionar procesos como el control tectónico del Valle de Toluca, su estratigrafía y los procesos paleogeomorfológicos que han influido en los procesos de sedimentación, comportamiento lacustre y posterior desecamiento de los cuerpos de agua, así como las modificaciones climáticas recientes, al igual que la dinámica y la evolución de las áreas industriales y los procesos de urbanización que han permitido la extracción extraordinaria del acuífero, más allá de los niveles pertinentes. 
Por principio de cuentas debe destacarse que el Valle de Toluca, al igual que el Valle de México, es una gran depresión tectónica, flanqueada por múltiples alineamientos tectónicos SE-NW y E-W, correspondientes en el primer caso a la Sierra de las Cruces y Monte Alto al Este, al igual que la Sierra del Campanario (o de Monte Alto) y el Nevado de Toluca al Oeste, mientras que para el segundo se puede mencionar a la Sierra de Tenango al Sur y a las elevaciones de la Falla de Perales al norte, que ocluyen parcialmente a la salida hidrológica del Río Lerma (Figura 5).

Fig. 5. Distribución Tectónica del Valle de Toluca

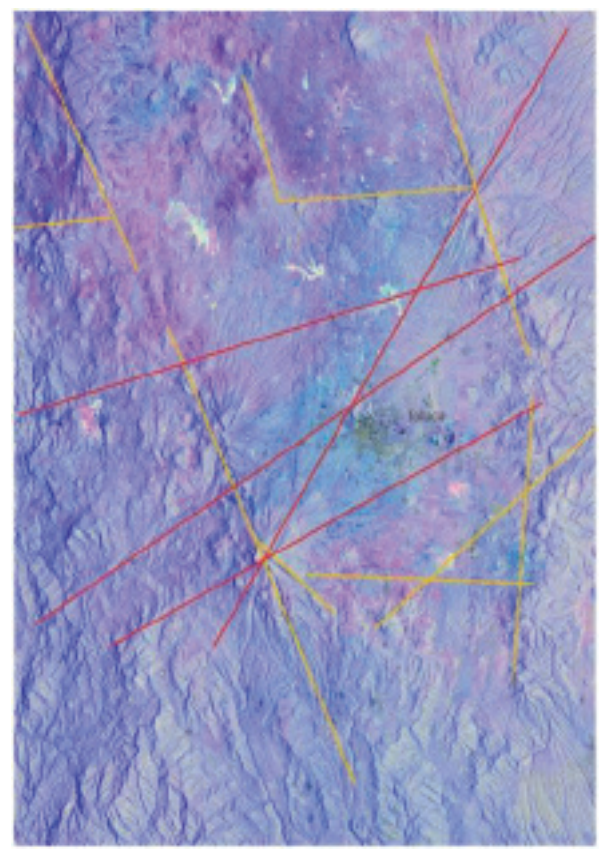

Fuente: Madrigal et al., 2010

Esta gran depresión es cruzada a su vez por tres grandes fallas tectónicas con orientación SW-NE, correspondientes a sizallamientos regionales (De Cserna, 1987; Hernández; 2007; Miranda; 2006) que han sido ampliamente estudiados y que influyen tanto en el comportamiento geohidrológico (Díez, 2006) a niveles profundos, como a niveles superficiales a través del basculamiento tectónico de vasos lacustres (Martínez, 1996; Madrigal et al., 2010). Además de estos grandes alineamientos tectónicos, existen muchas fallas menores y fracturamientos en todas las direcciones que se asocian a procesos de formación de campos de conos monoge- 
néticos al pie de las grandes sierras o como elevaciones o morros aislados dentro del valle, como los pequeños volcanes de las localidades de Tultepec, Ocoyacac, Atlapulco, San Miguel Almaya, Jalatlaco y San Nicolás Coatepec.

Toda esta actividad tectónica se asocia fuertemente con la actividad volcánica de la zona, que desde el periodo terciario hasta hace todavía 1200 años ha caracterizado toda la región, con todo tipo de manifestaciones volcánicas y con una gran diferenciación de materiales geológicos que se han acumulado en las laderas y el valle. Existen desde grandes estratovolcanes como el Nevado de Toluca donde dominan los materiales andesiticos y dacíticos, hasta campos de conos cineríticos, restos de avalanchas, pumitas y coladas recientes de lava basáltica.

Estos procesos, aunados a la incidencia de diferentes periodos glaciales, intercalados con periodos de resequedad, han generado sedimentos diversos que se han acumulado en la parte baja del valle de Toluca, reteniendo agua y humedad que dio origen a grandes lagos en forma basculada, separados entre sí por diques o depósitos fluvioglaciales, coladas y diferencias altitudinales marcadas por los sizallamientos tectónicos.

De esa manera se encontraba una laguna en la parte más alta, correspondiente al área que ocupa actualmente la Laguna de Chignahuapan y la Laguna de Almoloya del Río, la cual se vertía a través de un arroyo que cruzaba un dique natural dispuesto a la altura del casco de la Hacienda de Atenco (Santiago Tianguistenco) hacia la laguna siguiente.

La laguna intermedia ocupaba una mayor superficie que comprendía desde Tianguistenco y San Antonio la Isla hasta la localidades de Lerma, Mexicalcingo, Tultepec, Calpulhuac y San Mateo Atenco. Entre San Mateo Atenco y Lerma se volvía a formar un estrechamiento que estaba definido por antiguos sedimentos fluvioglaciales del Nevado y una gran colada de Lava en la porción $\mathrm{E}$.

El sistema hidro-lacustre terminaba con la formación de otra laguna más baja, que comenzaba en el estrechamiento mencionado y terminaba en las inmediaciones de Xonacatlán y Temoaya, al norte del valle. También existían otros cuerpos de agua separados de los grandes lagos, que correspondían a pequeñas cuencas divididas por la sierra de la Teresona y sus edificios asociados como los pequeños cerros de Coatepec, el Calvario y Metepec, dando lugar a pequeños vasos lacustres 
alimentados por los diferentes ramales del Río Verdiguel, que cruza en diferentes puntos a la ciudad de Toluca.

Todos estos cuerpos de agua fueron desecados, reducidos de tamaño 0 modificados por obras de infraestructura y el crecimiento de la mancha urbana e industrial, además de someter el acuífero a una demanda cada vez mayor de agua en su extracción, lo que ha generado zonas de subsidencia o hundimiento del terreno, tanto en zonas urbanas como en áreas rurales con actividades agropecuarias.

Particularmente el casco histórico de la Ciudad fue sometido hace 35 años a un proceso de extracción local de agua a base de 30 pozos que surtían del líquido a más de doce colonias alrededor del centro político de Toluca. No se había estudiado entonces que el manto freático local se encontraba sumamente superficial, confinado topográficamente y sujeto a un control estructural, dado por elevaciones volcánicas subterráneas, producto igualmente de morfoestructuras tectónicas locales.

El resultado de estas extracciones fue la de subsidencias sistemáticas que se manifestaron principalmente en la colonia Morelos, la colonia electricistas, plazas San Buenaventura y la colonia Granjas, a través de fracturamientos de las edificaciones, rupturas de tuberías y drenajes y agrietamiento de vialidades. Por la misma razón fueron clausurados los pozos locales y la red de agua potable se conectó a la distribución del Sistema Cutzamala, obra de gran impacto federal para abastecer de agua a la ciudad de México.

Los pozos de la zona industrial Toluca-Lerma siguieron funcionando al igual que los pozos de la periferia de la ciudad, independientemente de los aportes adicionales del Sistema Cutzamala, de modo que el abatimiento freático adquirió un ritmo acelerado que se manifesta en los datos presentados de este estudio y en las tres zonas seleccionadas para los interferogramas.

En las verificaciones de campo de las tres zonas se observan desniveles topográficos de hasta 2 metros de profundidad, grietas de entre $20 \mathrm{cms}$ y 1.7 metros, cambios en la red de drenaje superficial y subsuperficial, abatimiento de pozos artesianos, agrietamiento de viviendas y bardas y aumento de los niveles de contaminación del agua y del suelo por desechos industriales y domésticos, por efecto del confinamiento de las aguas residuales. En las zonas rurales, además de 
estos efectos se ha perdido el $40 \%$ de la humedad efectiva del suelo, debido a la extracción y el abatimiento.

\section{DISCUSIÓN Y CONCLUSIONES}

Contrariamente a los resultados de investigaciones anteriores en donde los niveles piezométricos no tenían una relación directamente proporcional con los datos aportados por el análisis de pares interferométricos, los resultados obtenidos a través del método de Persistent Scatterer sí demuestran una relación directamente proporcional con los datos piezométricos, la existencia de morfoestructuras tectónicas y los procesos de urbanización e industrialización, lo que proporciona una mayor eficacia en su aplicación a los procesos de subsidencia por sobreexplotación del acuífero.

Aunque muchos de los valores obtenidos son negativos y podrían desacreditar esta correspondencia, la diferencia en la velocidad de las subsidencias con relación al espectro de detección del sensor del radar simplemente establecen una correspondencia directa con los niveles piezométricos de las zonas de estudio, en particular en el casco histórico de la ciudad de Toluca, donde las velocidades del abatimiento del acuífero exceden con mucho la capacidad del sensor, pero delatan igualmente el proceso de hundimiento, independientemente de los valores negativos, que se interpretarían al revés.

El estudio constituye una primera aproximación al proceso, que se complementará con el registro sistemático y la cartografía de los efectos visibles de la subsidencia y la medición milimétrica de campo con estaciones robóticas totales de precisión topográfica, al igual que con mediciones magnetométricas y geofísicas, cuya implementación se encuentra ya en desarrollo a través de una nueva etapa de la investigación.

\section{REFERENCIAS BIBLIOGRÁFICAS}

- $\quad$ Calderhead, A; Martel, A; Alasset, P.J; Rivera, A; Garfias, J. "Land subsidence induced by groundwater pumping, monitored by D-InSAR and field data in the Toluca Valley, Mexico". Canadian Journal of Remote Sensing, v. 36, n. 1, p. 9-23. 2011. 
- $\quad$ Calderhead, A; Therrien, R; Rivera, A; Martel; Garfias, J. "Simulating pumping-induced regional land subsidence with the use of InSAR and field data in the Toluca Valley, Mexico". Advances in Water Resources, v. 34, n. 1, p. 83-97. 2010.

- Colesanti, C; Ferretti, A; Novali, F; Prati, C; Rocca, F. (2003). "SAR monitoring of progressive and seasonal ground deformation using the Permanent Scatterers Technique". IEEE Transactions on Geoscience and Remote Sensing 41(7):1685-1701.

- Díez, Pérez A. (2009). Alternativas de administración racional de recursos hídricos en un acuífero sometido a sobreexplotación: Valle de Toluca, Estado de México. Tesis de Doctorado en Ciencias del Agua. Facultad de Ingeniería de la UAEMEX, Toluca, Méx.terers Technique". IEEE Transactions on Geoscience and Remote Sensing 41(7):1685-1701.

- Díez, Pérez A. (2009). Alternativas de administración racional de recursos hídricos en un acuífero sometido a sobreexplotación: Valle de Toluca, Estado de México. Tesis de Doctorado en Ciencias del Agua. Facultad de Ingeniería de la UAEMEX, Toluca, Méx.

- Hernández, Quezada, E.R. (2007). Procesos Geomorfológicos en las vertientes del Municipio de Lerma, Méx.. Tesis de Licenciatura en Geografía. Facultad de Geografía de la UAEMEX, Toluca, Méx.

- Madrigal, U.D., R. Franco P., L.M. Espinosa R., M.A. González T. y A. Reyes R. (2010). "Caracterización de las Regiones Tectónicas del Estado de México a través de la aplicación de Geotecnologías". En Revista Geográfica de América Central. Vol. 1, Número 44 Especial 2010 (XII CONFIBSIG). Universidad Nacional de Costarrica, Costarrica. Pp. 15-36.

- Martínez, Calderón, Y.I. (1993). La Evolución de la Superficie de las Lagunas de Lerma y sus Principales Repercusiones Físicas, Biológicas y Humanas. Tesis de Licenciatura en Geografía. Facultad de Geografía de la UAEMEX, Toluca, Méx. 
- Miranda, Mendoza, M.R. (2006). Geomorfología de la Carta Jiquipilco 1:50 000 del INEGI. Tesis de Licenciatura en Geografía. Facultad de Geografía de la UAEMEX, Toluca, Méx.

- Werner, C; Wegmüller. U; Strozzi, T; Wiesmannn, A. "Interferometric point target analysis for deformation mapping". In: Geosciences and remote sensing symposium, Toulouse, France, 21-25 Jul 2003, pp 1-3 (CDROM).

- Sousa, J; Ruiz, A; Hanssen, R; Bastos, L; Gil, A; Galindo-Zaldívar, J;Galdeano, C. (2010). PS-InSAR processing methodologies in the detection of field surface deformation-Study of the Granada basin (Central Betic Cordilleras, southern Spain)

- Ferretti, A; Prati, C; Rocca, F. (2000). Analysis of Permanent Scatters in SAR Interferometry. Geoscience and Remote Sensing Symposium. Proceedings. IGARSS, 761-763.

- (Raucoles et al., 2003; Croseto et al., 2005; Akcin et al., 2010; Honh Hong et al., 2010 ; Osmanoglu et al., 2011; Cigna et al., 2011 ; Rodríguez et al., 2012;

- $\quad$ Chen, F; Lin, H; Zhang, Y; Lu, Z. 2012. Ground subsidence geo-hazards induced by rapid urbanization: implications from InSAR observation and geological analysis; Natural Hazards and Earth System Sciences; 12, 935-932.

- Herrera, G; Fernandez, R; Tomás, R, Cooksley, G; Mulas, J. 2009. Advanced interpretation of subsidence in Murcia (SE Spain) using A-DInSAR data-modelling validation; Natural Hazards and Earth System Sciences, 647-661.

- $\quad$ López-Quiroz, P; Marie-Pierre, D; Tupin F; Briole, P; Jean-Marie, N. 2009. Time series analysis of Mexico City subsidence constrained by radar interferometry; Journal of Applied Geophysics; 69; 1-15.

- Gárfias, J; Bibiano, L; Llanos, H. (2008). Uso racional y sostenible de los recursos hídricos del acuífero del valle de Toluca; Ciencia Ergo Sum, vol. 15, núm. 1, marzo-junio, pp. 61-72, Universidad Autónoma del Estado de México. 